\title{
Investigating the origin of third harmonic generation from diabolo optical antennas
}

\author{
Liping Shi, ${ }^{1,2, a)}$ José R. C. Andrade, ${ }^{1,2}$ Hyunwoong Kim, ${ }^{3}$ Seunghwoi Han, ${ }^{3}$ Rana Nicolas, ${ }^{4}$ \\ Dominik Franz, ${ }^{4}$ Willem Boutu, ${ }^{4}$ Torsten Heidenblut, ${ }^{5}$ Frans B. Segerink, ${ }^{6}$ Bert Bastiaens, ${ }^{7}$ \\ Hamed Merdji, ${ }^{4}$ Seung-Woo Kim, ${ }^{3}$ Uwe Morgner, ${ }^{1,2}$ and Milutin Kovačev ${ }^{1,2}$ \\ ${ }_{1}^{1}$ Institut für Quantenoptik, Leibniz Universität Hannover, Welfengarten 1, 30167 Hannover, Germany \\ ${ }^{2}$ QUEST, Centre for Quantum Engineering and Space-Time Research, 30167 Hannover, Germany \\ ${ }^{3}$ Department of Mechanical Engineering, Korea Advanced Institute of Science and Technology (KAIST), \\ Science Town, Daejeon 305-701, South Korea \\ ${ }_{5}^{4}$ LIDYL, CEA, CNRS, Université Paris-Saclay, CEA Saclay 91191 Gif-sur-Yvette, France \\ ${ }^{5}$ Institut für Werkstoffkunde, Leibniz Universität Hannover, An der Universität 2, 30823 Garbsen, Hannover, \\ Germany \\ ${ }^{6}$ Optical Sciences, MESA+Institute for Nanotechnology, University of Twente, P.O. Box 217, 7500AE \\ Enschede, The Netherlands \\ ${ }^{7}$ Laser Physics and Nonlinear Optics, Mesa+ Institute for Nanotechnology, University of Twente, 7500AE \\ Enschede, The Netherlands
}

(Received 21 August 2017; accepted 9 October 2017; published online 24 October 2017)

\begin{abstract}
We propose to use diabolo nanoantennas for experimentally investigating the origin of the enhanced third harmonic generation by localized surface plasmon polaritons. In such a geometry, the opposing apexes of bowties are electrically connected by a thin gold nanorod, which has two important functions in discriminating the point of harmonic generation. First, the inserted gold nanorod shifts the field enhancement area to be far away from the dielectric substrate material. Next, the accumulation of free charges at the adjacent bowtie tips produces a strong electric field inside the gold nanorod. The diabolo nanoantennas allow us to examine the contribution of the bare gold susceptibility to the third harmonic conversion. Our results reveal that the bare gold does not significantly enhance the harmonic generation at high pump intensity. From this, we deduce that in regular bowtie antennas, the enhanced harmonic photons mainly arise from the substrate sapphire that is located in the feedgap of the bowtie, where the electric near-field is significantly enhanced by the localized surface plasmons. Published by AIP Publishing. https://doi.org/10.1063/1.5001005
\end{abstract}

By confining the incoming light to a deep-subwavelength area, plasmonic nanoantennas can be employed to control and manipulate optical fields at the nanoscale. ${ }^{1-4}$ In recent years, optical antennas have attracted considerable interest and hold promise to improve the performance in a large number of applications, such as near-field scanning optical microscopy, ${ }^{5}$ photo-detectors, ${ }^{6}$ photovoltaic cells, ${ }^{7}$ thermal emitters, ${ }^{8}$ saturable absorbers, ${ }^{9}$ upconverted incoherent nonlinear emission, ${ }^{10,11}$ single-molecule fluorescence enhancement, ${ }^{12}$ and third harmonic generation (THG) enhancement. ${ }^{13}$ Due to the lightning-rod effect and the coupling of two metal nanotriangles (coupled plasmons), it has been shown that bowtie nanoantennas ${ }^{14}$ can achieve stronger intensity enhancement than other comparable structures such as rod-, ellipse-, disk-, and cross-shaped nanoantennas. ${ }^{15,16}$ Therefore, they have been proposed to boost the field intensity of pulses from femtosecond oscillators to assist extremely nonlinear optics, such as the generation of a deep-ultraviolet light source. ${ }^{17}$ The surface plasmon enhanced harmonic conversion holds the potential for applications in nonlinear spectroscopy and quantum optics through the manipulation of light on a subwavelength scale.

In order to more efficiently convert light at the nanoscale, the hybrid plasmonic-dielectric nanoantennas have been proposed, which consist of dimeric gold ( $\mathrm{Au}$ ) antennas with a high-nonlinearity dielectric crystal. ${ }^{18}$ Despite the fact

a)shi@iqo.uni-hannover.de that the enhancement of harmonic conversion by the hybrid nanostructures is extensively observed, the origin of the boosted signal is still controversial. ${ }^{18,19}$ The harmonic enhancement, on the one hand, may arise from the nonlinear polarization of the crystal within the optical hot spot, yet, on the other hand, it could also directly originate from the bare $\mathrm{Au}$ itself. Recently, the origin of THG enhancement is mainly distinguished via the shift of harmonic spectra by hybrid dipole nanoantennas. ${ }^{20-22}$

In this letter, by investigating the dependence of the harmonic intensity on the polarization of the laser, we demonstrate another method to determine the origin of the THG by dipole nanoantennas. Instead of positioning a dielectric crystal into the feedgap, here we insert a thin Au nanorod to electrically connect the adjacent bowtie nanoantenna tips, which is referred to as the interconnector hereafter. Under the illumination of a broadband femtosecond laser polarized along the antenna long-axis, a strong electric field is induced inside the interconnector due to the accumulation of free charges at the opposite tips. In case the THG would mainly arise from the nonlinear polarization of $\mathrm{Au}$ atoms, one can expect a significant signal enhancement due to the field enhancement inside the $\mathrm{Au}$ nanorod. Here, the THG enhancement is defined as the intensity ratio when the laser is polarized parallel with respect to the case that the laser is polarized perpendicular to the antenna long-axis. However, if the THG would dominantly originate from the optical hotspot at the 

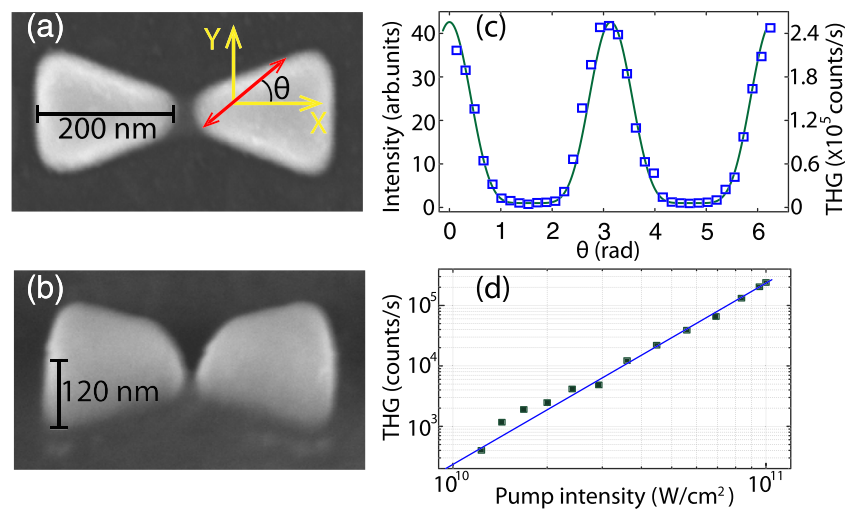

FIG. 1. SEM images of a gold bowtie nanoantenna: (a) top view and (b) perspective view. The double arrow indicates the direction of laser polarization. The crossing angle between the laser polarization and long-axis of the dimeric antenna, i.e., $\mathrm{X}$-axis, is defined as $\theta$. (c) The dependence of $\mathrm{TH}$ radiation from the bowtie antenna on $\theta$. (d) THG intensity (squares) as a function of pump intensity when the laser polarized along the antenna long-axis, i.e., $\theta=0$. The $\mathrm{TH}$ photon counts scales with the cube (blue line) of the pump intensity.

substrate surface, there will be no significant harmonic amplification because emission in the gap region is hindered by the Au interconnector.

First, we measure the dependence of THG on the direction of laser polarization by using the regular bowtie antennas, i.e., a pair of tip-to-tip triangles. Figures 1(a) and 1(b) show the top- and perspective-view scanning electron microscopy (SEM) images of a representative bowtie specimen that was fabricated by focused-ion-beam (FIB) milling on a sapphire $\left(\mathrm{Al}_{2} \mathrm{O}_{3}\right)$ substrate covered with an $\mathrm{Au}$ layer. The substrate has a thickness of $400 \mu \mathrm{m}$. The length, thickness, apex angle, and curvature radius of a single $\mathrm{Au}$ triangle nanostructure are $200 \mathrm{~nm}, 120 \mathrm{~nm}, 30^{\circ}$, and $20 \mathrm{~nm}$ respectively. The gap distance of the bowtie is $\sim 20 \mathrm{~nm}$. These nanoantennas were arranged in square arrays of $12 \mu \mathrm{m} \times 12 \mu \mathrm{m}$, with a spacing of $500 \mathrm{~nm}$ in the $x$ direction and $200 \mathrm{~nm}$ in the $y$ direction. The femtosecond pulses from a mode-locked Ti:sapphire oscillator centered at $800 \mathrm{~nm}$ (spectrum ranging from 650 to $1000 \mathrm{~nm}$ ), with a repetition rate of $100 \mathrm{MHz}$ and a duration of $8 \mathrm{fs}$, are tightly focused by an off-axis parabolic mirror onto a bowtie array. The focal spot of the incident laser on the nanostructures has a diameter of $\sim 7 \mu \mathrm{m}$, resulting in the simultaneous emission of third harmonic $(\mathrm{TH})$ from approximately 250 of the individual antennas. The pump intensity on the nanoantennas is $10^{11} \mathrm{~W} / \mathrm{cm}^{2}$, slightly below the damage threshold. ${ }^{23} \mathrm{We}$ employ a broad bandwidth half-wave plate to rotate the laser polarization. The generated $\mathrm{TH}$ photons propagating in the forward direction are refocused by a toroidal grating into a photomultiplier, which is combined with a photon counter for detection. The acceptance angle of the detection system is $17^{\circ}$.

Figure 1(c) depicts the intensity of TH (open squares) emission from a bowtie array versus the crossing angle $(\theta)$ between the laser polarization and the long axis of the antennas ( $x$-axis). The photon counts are normalized to the signal when $\theta=\pi / 2$. The THG intensity scales with the cube of the driver intensity [Fig. 1(d)], $I_{3 \omega} \propto I_{\omega}{ }^{3} \propto\left(E_{\omega} \cdot \cos \theta\right)^{6}$. The measured TH counts can be excellently fitted by $\cos ^{6} \theta$ [Fig. 1(c), solid curve]. From Fig. 1(c), one can see that the THG is amplified by a factor of $>40$ when the laser is polarized along the antenna long-axis. However, we cannot unveil the origin of this enhanced harmonic, as it may radiate from two different sources: (i) the nonlinear polarization of $\mathrm{Al}_{2} \mathrm{O}_{3}$ surrounding the bowtie tips and (ii) the nonlinear polarization of the bare Au antennas.

Using the open source of software package MEEP, ${ }^{24}$ we perform the nonlinear finite-difference time-domain (FDTD) simulation of $\mathrm{TH}$ emission from the Au antennas and from the $\mathrm{Al}_{2} \mathrm{O}_{3}$ substrate. The third-order susceptibilities of $\mathrm{Au}$ and $\mathrm{Al}_{2} \mathrm{O}_{3}$ are set to be $\chi^{(3)}(\mathrm{Au}) \sim 10^{-19} \mathrm{~m}^{2} / \mathrm{V}^{2}$ (Ref. 25) and $\chi^{(3)}$ $\left(\mathrm{Al}_{2} \mathrm{O}_{3}\right) \sim 10^{-22} \mathrm{~m}^{2} / \mathrm{V}^{2}$ (Ref. 26), respectively. A Gaussian pulse with the central wavelength of $800 \mathrm{~nm}$ and a pulse duration of $8 \mathrm{fs}$ is employed as the pump source. Figure 2 shows the results of numerical simulation. Compared to the pump light polarized at $\theta=\pi / 2$ (blues curves), the intensities of $\mathrm{TH}$ emission both from the bare Au antenna [Fig. 2(a)] and from the $\mathrm{Al}_{2} \mathrm{O}_{3}$ substrate [Fig. 2(c)] are evidently amplified when $\theta=0$ (red curves). This is in agreement with the linear FDTD simulation of near-field distribution. As can be seen from the insets in Figs. 2(a) and 2(c), at $\theta=0$, the field strengths both in $\mathrm{Au}$ and in $\mathrm{Al}_{2} \mathrm{O}_{3}$ are much stronger with respect to $\theta=\pi / 2$.

It should be pointed out that the electric field $E(r)$ inside Au reaches its highest strength at the shank rather than at the tips of the bowtie [left inset, Fig. 2(a)]. This is because the electric field inside the metal is proportional to the plasmonic current density, which peaks in the region where the charges can freely flow, namely, in the center region of the Au nanostructures. Therefore, the polarization intensity $P(r)$ in $\mathrm{Au}$ is also linear with the plasmonic current density $j(r)$, i.e., $P(r) \propto \varepsilon_{0}\left(\varepsilon_{m}-1\right) E(r) \propto j(r) / \omega$, where $\varepsilon_{o}, \omega$, and $\varepsilon_{m}$ are the vacuum permittivity, laser angular frequency, and permittivity of the metal, respectively. When a thin Au interconnector is positioned between the adjacent bowtie apexes, the Au triangles act as an electric funnel which reinforces the optical current density into this interconnector when the laser is polarized along the antenna's long-axis. ${ }^{27}$ The flow of numerous free electrons within this small volume induces a high field strength and thus a stronger plasmonic current inside the Au nanorod [left inset, Fig. 2(b)]. The Au interconnector shifts the field enhancement area to be far away from the substrate. ${ }^{28}$ There is no field enhancement in the substrate of the gap region [left inset, Fig. 2(d)]. Hence, there is no possibility for the gap-sapphire to radiate harmonics. If the THG emission from $\mathrm{Au}$ is dominant, by using the interconnector, we should observe an even higher $\mathrm{TH}$ photon counts at $\theta=0$ due to the enhancement of field inside $\mathrm{Au}$ [red curve, Fig. 2(b)]. Otherwise, the THG should be much lower and its intensity is expected to have a weaker dependence on the laser polarization [Fig. 2(d)], as the field inside the $\mathrm{Al}_{2} \mathrm{O}_{3}$ substrate in the gap region is always suppressed by the Au nanorod [insets, Fig. 2(d)].

In order to experimentally explore the proposed system, we fabricated another array of nanoantennas with identical parameters as the aforementioned bowtie but adding an $\mathrm{Au}$ interconnector into the gap. This shape is named the diabolo nanoantenna in the literature. ${ }^{29}$ Figures 3(a) and 3(b) show the SEM images of a representative diabolo in top and perspective views (titled angle $45^{\circ}$ ). It can be seen that the bowtie feedgap is connected by an Au nanorod with a width of $\sim 30 \mathrm{~nm}$ and a thickness of $\sim 35 \mathrm{~nm}$. Figures $3(\mathrm{c})$ and $3(\mathrm{~d})$ 

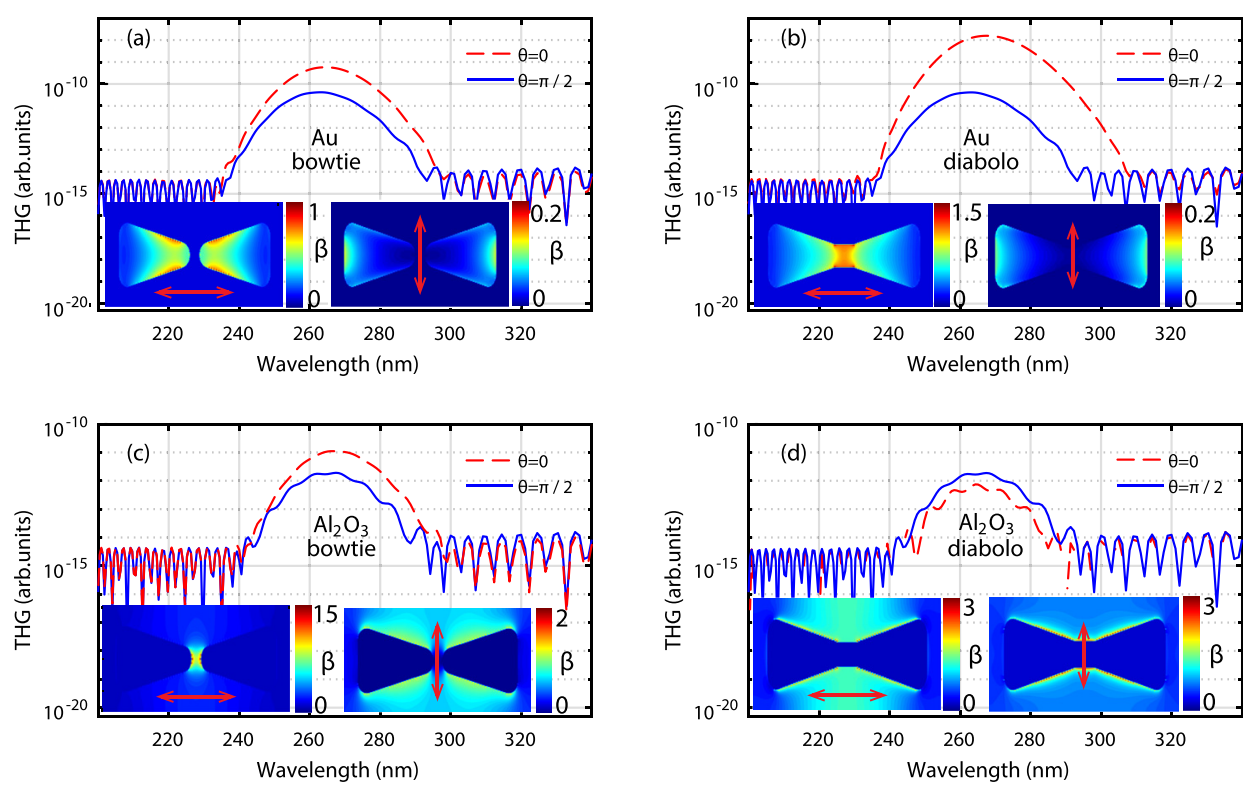

FIG. 2. Nonlinear FDTD simulation of THG solely emitted from the bare gold antenna (a) and (b) and from the sapphire substrate (c) and (d). The dashed red $(\theta=0)$ and solid blue $(\theta=\pi / 2)$ curves corresponding to the polarizations of the laser are parallel and perpendicular to the long-axis of antennas, respectively. For diabolo antennas (b), the presence of a gold nanorod in the gap leads to a stronger TH emission with respect to the bowtie one (a). Insets: (a) At the central wavelength of $800 \mathrm{~nm}$, electric distribution inside gold ( $z=118 \mathrm{~nm}$, i.e., $2 \mathrm{~nm}$ below the air-bowtie interface) when the laser polarization is parallel (left inset) and perpendicular (right inset) to the long-axis of the bowtie antenna is shown. (b) Electric distribution inside gold ( $z=33 \mathrm{~nm}$, i.e., $2 \mathrm{~nm}$ below the air-nanorod interface) when the laser polarization is parallel (left inset) and perpendicular (right inset) to the long-axis of the diabolo antenna. (c) Electric distribution inside the substrate $(z=-2 \mathrm{~nm}$, i.e., $2 \mathrm{~nm}$ below the bowtie-sapphire interface) when the laser polarization is parallel (left inset) and perpendicular (right inset) to the bowtie antenna. (d) Electric distribution inside the substrate $(z=-2 \mathrm{~nm}$, i.e., $2 \mathrm{~nm}$ below the bowtie-sapphire interface) when the laser polarization is parallel (left inset) and perpendicular (right inset) to the diabolo antenna. Here, $\beta$ denotes the electric field enhancement factor, i.e., $\left|E / E_{0}\right|$.

display the side-view near-field distribution of the bowtie and diabolo antenna, respectively. As mentioned above, the presence of an Au nanorod in the gap [Fig. 3(d)] pushes the hotspot [Fig. 3(c)] to be far away from the substrate. We repeat the measurement of THG intensity as a function of laser polarization using this diabolo antenna array, as plotted in Fig. 3(e). We find that the maximum THG is only enhanced by a factor of 2.5 , which suggests that there might exist slight enhancement of THG by bare Au. Nevertheless, the THG enhancement factor of diabolo antennas is evidently lower than that of a bowtie array [Fig. 1(c)]. To further compare the polarization dependent enhancement factor of THG emission from the bowtie and the diabolo, Fig. 3(g) plots the absolute value of TH photon counts in a polar diagram. It is clearly shown that the THG emission from the diabolo configuration (red circles) is significantly lower than its regular counterpart (blue squares). This result suggests that the bare $\mathrm{Au}$ does not contribute to the far-field $\mathrm{TH}$ radiation as significant as the simulations expected [Figs. 2(a) and 2(b)].

To further understand the radiation's origin, we perform a $z$-scan measurement. The inset of Fig. 4(a) illustrates the experimental approach. The laser propagates from left to right. As a reference, Fig. 4(a) plots the counts of TH radiation from the bare $\mathrm{Al}_{2} \mathrm{O}_{3}$ substrate as a function of the focal position. Two pronounced maxima appear with a spatial separation of $400 \mu \mathrm{m}$, which exactly equals the thickness of our substrate. The lower THG emission from the back (right) surface with respect to the front (left) one is caused by an increased pulse duration due to the propagation of the laser through the dispersive substrate. Figure 4(b) shows the photon counts of the TH emission from a regular bowtie array versus focal position. The laser is polarized along the $x$-axis. In strong contrast to the bare substrate, we find that the THG radiated from the back surface which is nanostructured with bowties is drastically amplified. Meanwhile, compared to the THG spectral emission from the bare substrate, an apparent blue shift is observed, as shown in the right inset of Fig. 3(b). This can be attributed to the shift of the field enhancement wavelength with respect to the central wavelength of the laser. The right inset of Fig. 4(c) shows the spectra of TH radiated from diabolo antennas and bare substrate. Also, here we observe a slight blue shift. From the $z$-scan result of the diabolo array [Fig. 4(c)], an amplification of THG from the back surface is also found. However, its enhancement factor with respect to the front surface is one order of magnitude lower than that of the bowtie array. This comparison further suggests that the $\mathrm{Au}$ interconnector might contribute to the THG enhancement, but its yield efficiency is much weaker than that of the dielectric substrate which is located at the optical hotspot of bowtie feedgap. Furthermore, we find that the photon counts of $\mathrm{TH}$ emission from the bare $\mathrm{Al}_{2} \mathrm{O}_{3}$ surface [Fig. 4(a)] are almost on the same order of magnitude with respect to the nanostructured surface [Fig. 4(c)]. However, the nonlinear FDTD simulations indicate that if the THG would mainly originate from $\mathrm{Au}$, the antennas [Figs. 2(a) and 2(b)] should produce several orders of magnitude higher THG with respect to the bare $\mathrm{Al}_{2} \mathrm{O}_{3}$ crystal [Figs. 2(c) and 2(d)]. Consequently, from the comparison of photon counts, we can further conclude that the bare $\mathrm{Au}$ does not significantly contribute to the THG enhancement.

One might ask whether a strong electric field indeed exists within the $\mathrm{Au}$ interconnector of the diabolo antennas. To verify the enhancement of the electric field inside the 


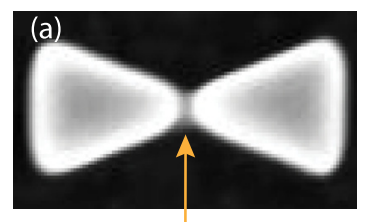

Au nanorod
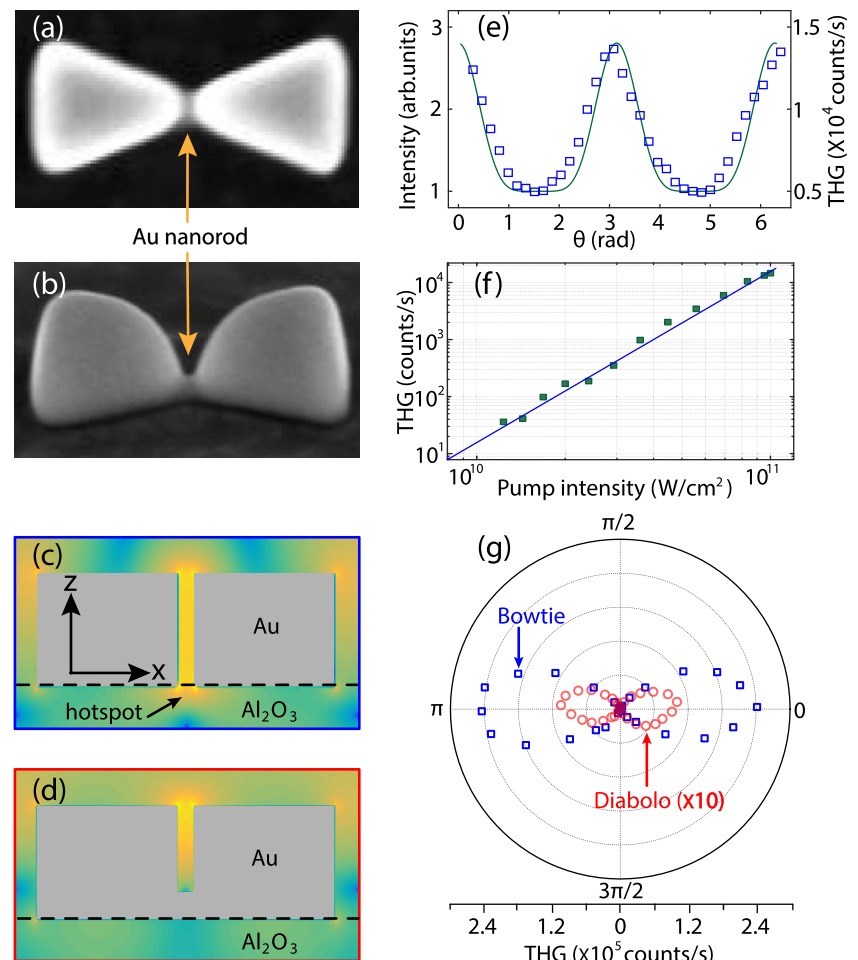

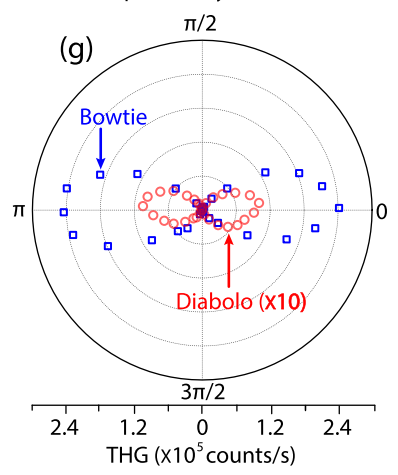

FIG. 3. Top (a) and perspective (b) view SEM images of a diabolo antenna. A thin gold nanorod electrically connected the facing tips. The side-view (at $y=0$ ) electric near-field distribution of the bowtie (c) and diabolo (d) antenna is shown. The laser propagates along the $z$-axis and polarizes along the $x$-axis. The interface (dashed lines) between the Au nanoantenna and the $\mathrm{Al}_{2} \mathrm{O}_{3}$ substrate is defined as $z=0$. The comparison between (c) and (d) shows that the presence of the gold nanorod in the diabolo antennas pushes the hotspot to be far away from the substrate. (e) and (f) Photon counts of $\mathrm{TH}$ emission from diabolo antennas as a function of $\theta$ (e) and pump intensity (f): the TH photon counts scale with the cube (blue line) of the pump intensity. (g) Counts of TH emission from the bowtie (blue squares) and diabolo (red circles) antennas versus laser polarization in the polar diagram.

interconnector, we utilize the photothermal effects. ${ }^{30}$ Thermal heating in plasmonic nanoantennas is governed by ohmic heating, with the heat source density given by $q=\frac{1}{2} \varepsilon_{0} \omega \operatorname{Im}\left(\varepsilon_{m}\right)|E(r)|^{2}=|j(r)|^{2} / \sigma$, where $\sigma$ denotes the electrical conductivity of Au. Here, $|E(r)|$ is the electric field strength and $|j(r)|$ is the plasmonic current density inside the metal. The plasmonic nanoparticles can be thermally reshaped when the metal is heated to a critical point by the current. Figure 5 shows a side-view SEM image of a diabolo antenna after long-term irradiation by high-fluence femtosecond pulses $\left(30 \mathrm{~J} / \mathrm{m}^{2}\right)$. The side-view SEM image is obtained by milling a cross section of the nanostructure in the $x-z$ plane with a $\mathrm{Ga}^{+}$-based FIB. From the SEM image, we can clearly observe that the $\mathrm{Au}$ interconnector is destroyed, resulting in the separation of a fragment due to the change in surface tension at high temperature. This structural deformation provides an important evidence that, indeed, a local plasmonic current passes through the Au interconnector, and a strong electric field was induced inside the Au nanorod.

Regardless of the huge third order nonlinear susceptibility of Au nanostructures, our measurement however clearly confirms that the THG mainly radiates from the exposed sapphire in the optical hot spot of the bowtie, namely, in the antenna gap region. The absence of THG from the bare Au may be attributed to the following two reasons: First, in our
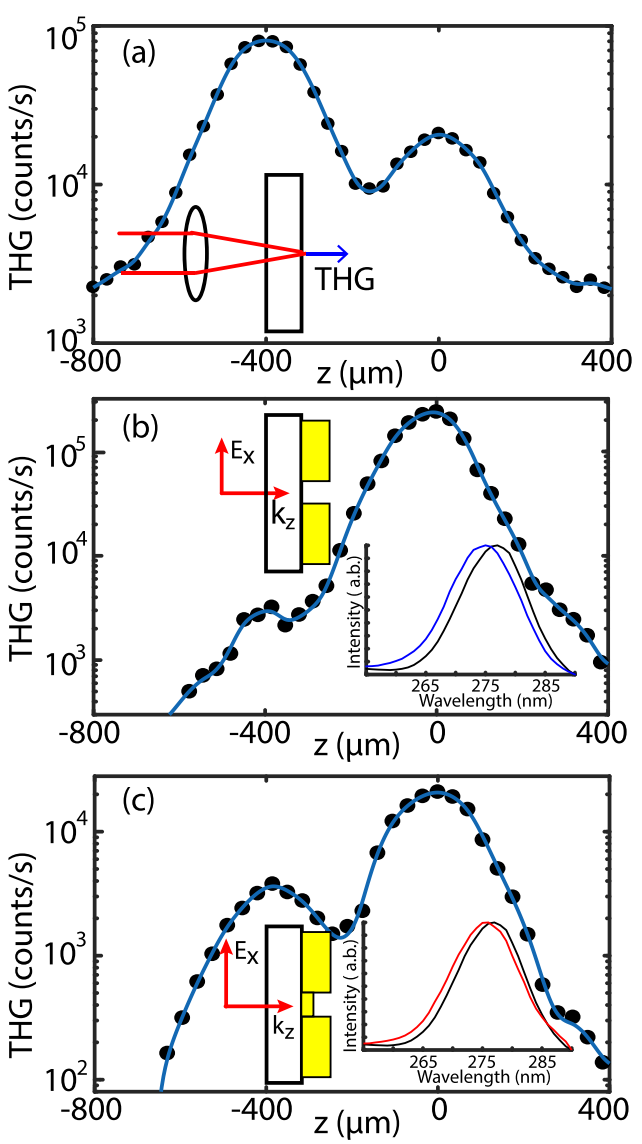

FIG. 4. (a) Photon counts of TH emission from the bare substrate surface versus the laser focus position. The inset shows the experimental setting from a side view. The laser propagates from left to right, i.e., along the $z$ axis. The back (left) surface corresponds to the position of $-400 \mu \mathrm{m}$. (b) zscan of $\mathrm{TH}$ emission from bowtie antennas, which are located at the front (right) surface of the substrate and laser polarization along the $x$-axis. The left inset shows the spectra of THG radiated from the bare substrate surface (black curve) and from the bowtie surface (blue curve). (c) z-scan of TH emission from diabolo antennas. The left inset shows the spectra of THG radiated from the bare substrate surface (black curve) and from the bowtie surface (red curve).

experimental conditions, the interband transition of $\mathrm{Au}$ atoms becomes significant at the rather high pump intensity. The photon energy of our femtosecond oscillator is $\hbar \cdot \omega=1.55 \mathrm{eV}$, and therefore, two-photon absorption $(3.1 \mathrm{eV})$ is already more than enough to excite electrons from the $5 d$-bands crossing the Fermi surface and entering the empty states in the 6spbands $\left(\Delta E=E_{6 s p}-E_{5 d} \sim 2.2 \mathrm{eV}\right){ }^{31}$ The dominance of twophoton band-to-band absorption can restrain the real part of the third-order nonlinear response of materials, which therefore quenches the THG conversion efficiency. ${ }^{31}$ Second, the energy of a single THG photon in our case reaches $\hbar \cdot 3 \omega=4.65 \mathrm{eV}$. This energetic photon can be reabsorbed by the Au atoms through the electron-electron scattering assisted absorption and the interband absorption. ${ }^{32-34}$ The strong reabsorption further significantly prevents the transmission of a THG signal.

In summary, we propose the use of diabolo nanoantennas to investigate the origin of enhanced THG emitted from dipolar plasmonic nanostructures. Our experimental results demonstrate that the bare $\mathrm{Au}$ atoms might contribute to the THG emission; however, the exposed dielectric substrate in the gap region of an $\mathrm{Au}$ antenna dominates the THG 


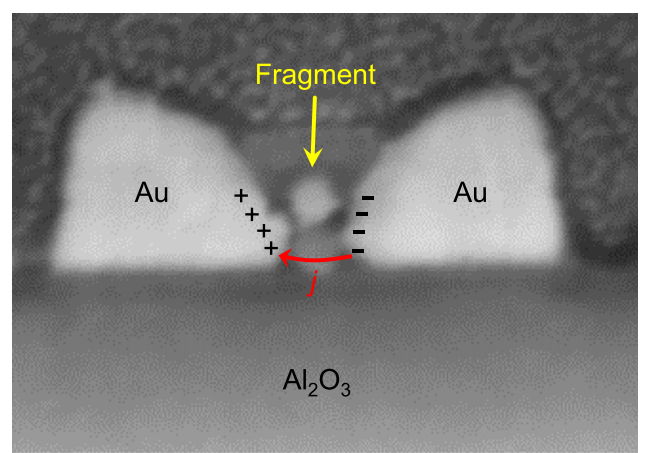

FIG. 5. SEM image of a diabolo nanoantenna after a long-term irradiation by intense pulses. The accumulation of free charges at the neighbouring tips results in a strong plasmonic current (red curve) passing through the small volume of the interconnector. This highly concentrated current can contribute a thermal load to the gold nanorod through the ohmic heating process, which finally thermally destroys the gold interconnector. A separation of the fragment caused by thermal damage is observed in the gap, as indicated by the yellow arrow.

enhancement. Our results are in agreement with the recent plasmonic enhanced high harmonic generation from a crystalline material. $^{28,35}$ We do not observe a significant enhancement of the THG emission from the bare Au itself, which is ascribed to the strong absorption by Au atoms. It will be promising to use mid-infrared femtosecond sources to further investigate the ability of bare $\mathrm{Au}$ for $\mathrm{THG}$ enhancement because in the long wavelength region, the band-to-band transition is not as significant as in the case of a near-infrared driver.

We would like to thank the funding support from the Deutsche Forschungsgemeinschaft (DFG) under grant number KO 3798/4-1, from the Centre for Quantum Engineering and Space-Time Research (QUEST), from Lower Saxony through "Quanten-und Nanometrologie" (QUANOMET, project Nanophotonik), from the National Research Foundation of the Republic of Korea (NRF2012R1A3A1050386), from the ANR under the Grant IPEX (2014), and from the LABEX PALM (ANR-10-LABX0039) under the Grants Plasmon-X (2014) and HILAC (2015).

${ }^{1}$ L. Novotny and N. Van Hulst, Nat. Photonics 5, 83 (2011).

${ }^{2}$ J. Kim, A. Dutta, G. V. Naik, A. J. Giles, F. J. Bezares, C. T. Ellis, J. G. Tischler, A. M. Mahmoud, H. Caglayan, O. J. Glembocki, A. V. Kildishev, J. D. Caldwell, A. Boltasseva, and N. Engheta, Optica 3, 339 (2016).

${ }^{3}$ J. A. Schuller, E. S. Barnard, E. S. Cai, Y. C. Jun, J. S. White, and M. L. Brongersma, Nat. Mater. 9, 193 (2010).

${ }^{4}$ D. Smirnova and Y. S. Kivshar, Optica 3, 1241 (2016).
${ }^{5}$ T. H. Taminiau, R. J. Moerland, F. B. Segerink, L. Kuipers, and N. F. Van Hulst, Nano Lett. 7, 28 (2007).

${ }^{6} \mathrm{M}$. W. Knight, H. Sobhani, P. Nordlander, and N. J. Halas, Science 332, 702 (2011).

${ }^{7}$ M. L. Brongersma, Y. Cui, and S. Fan, Nat. Mater. 5, 451 (2014).

${ }^{8}$ J. A. Schuller, T. Taubner, and M. L. Brongersma, Nat. Photonics 3, 658 (2009).

${ }^{9}$ J. Y. Suh, M. D. Huntington, C. H. Kim, W. Zhou, M. R. Wasielewski, and T. W. Odom, Nano Lett. 12, 269 (2012).

${ }^{10}$ K. Schraml, A. Regler, J. Bartl, G. Glashagen, J. Wierzbowski, J. J. Finley, and M. Kaniber, Optica 3, 1453 (2016).

${ }^{11}$ M. Sivis, M. Duwe, B. Abel, and C. Ropers, Nat. Phys. 9, 304 (2013).

${ }^{12}$ A. Kinkhabwala, Z. F. Yu, S. H. Fan, Y. Avlasevich, K. Mullen, and W. E. Moerner, Nat. Photonics 3, 654 (2009).

${ }^{13}$ H. Aouani, M. Rahmani, M. Navarro-Cía, and S. A. Maier, Nat. Nanotechnol. 9, 290 (2014).

${ }^{14}$ P. J. Schuck, D. P. Fromm, A. Sundaramurthy, G. S. Kino, and W. E. Moerner, Phys. Rev. Lett. 94, 017402 (2005).

${ }^{15}$ T. Hanke, J. Cesar, V. Knittel, A. Trugler, U. Hohenester, A. Leitenstorfer, and R. Bratschitsch, Nano Lett. 12, 992 (2012).

${ }^{16}$ E. Cubukcu, N. Yu, E. J. Smythe, L. Diehl, K. B. Crozier, and F. Capasso, IEEE J. Sel. Top. Quantum Electron. 14, 1448 (2008).

${ }^{17}$ S. Kim, J. H. Jin, Y. J. Kim, I. Y. Park, Y. Kim, and S. W. Kim, Nature 453, 757 (2008).

${ }^{18}$ H. Linnenbank, Y. Grynko, J. Förstner, and S. Linden, Light: Sci. Appl. 5, e16013 (2016).

${ }^{19}$ B. Metzger, M. Hentschel, T. Schumacher, M. Lippitz, X. Ye, C. B. Murray, B. Knabe, K. Buse, and H. Giessen, Nano Lett. 14, 2867 (2014).

${ }^{20}$ H. Aouani, M. Rahmani, M. Navarro-Cía, and S. A. Maier, Adv. Opt. Mater. 3, 986 (2015).

${ }^{21}$ D. de Ceglia1, M. A. Vincenti1, and M. Scalora, J. Opt. 18, 115002 (2016).

${ }^{22}$ A. C. Lesina, P. Berin, and L. Ramunno, Opt. Mater. Express 7, 1575 (2017).

${ }^{23}$ L. P. Shi, B. Iwan, R. Nicolas, Q. Ripault, J. R. C. Andrade, S. Han, H. Kim, W. Boutu, D. Franz, T. Heidenblut et al., Optica 4, 1038 (2017).

${ }^{24}$ A. F. Oskooi, D. Roundy, M. Ibanescu, P. Bermel, J. Joannopoulos, and S. G. Johnson, Comput. Phys. Commun. 181, 687 (2010).

${ }^{25}$ J. Renger, R. Quidant, N. van Hulst, and L. Novotny, Phys. Rev. Lett. 104, 046803 (2010).

${ }^{26}$ T. Utikal, T. Zentgraf, T. Paul, C. Rockstuhl, F. Lederer, M. Lippitz, and H. Giessen, Phys. Rev. Lett. 106, 133901 (2011).

${ }^{27}$ X. Xiong, Z. Xue, C. Meng, S. Jiang, Y. Hu, R. Peng, and M. Wang, Phys. Rev. B 88, 115105 (2013).

${ }^{28}$ S. Han, H. Kim, Y. W. Kim, Y. J. Kim, S. Kim, I. Y. Park, and S. W. Kim, Nat. Commun. 7, 13105 (2016).

${ }^{29}$ T. Grosjean, M. Mivelle, F. I. Baida, G. W. Burr, and U. C. Fischer, Nano Lett. 11, 1009 (2011).

${ }^{30}$ Z. J. Coppens, W. Li, D. G. Walker, and J. G. Valentine, Nano Lett. 13, 1023 (2013).

${ }^{31}$ T. Hanke, G. Krauss, D. Träutlein, B. Wild, R. Bratschitsch, and A. Leitenstorfer, Phys. Rev. Lett. 103, 257404 (2009).

${ }^{32}$ J. B. Khurgin, Nat. Nanotechnol. 10, 2 (2015).

${ }^{33}$ D. de Ceglia, M. A. Vincenti, N. Akozbek, M. J. Bloemer, and M. Scalora, Opt. Express 25, 3980 (2017).

${ }^{34}$ M. A. Vincenti, D. de Ceglia, C. de Angelis, and M. Scalora, J. Opt. Soc. Am. B 34, 633 (2017).

${ }^{35}$ G. Vampa, B. G. Ghamsari, S. Siadat Mousavi, T. J. Hammond, A. Olivieri, E. Lisicka-Skrek, A. Y. Naumov, D. M. Villeneuve, A. Staudte, P. Berini, and P. B. Corkum, Nat. Phys. 13, 659 (2017). 